\title{
CD34 is required for the infiltration of inflammatory cells into the mouse colon during DSS-induced colitis
}

\author{
Steven Maltby ${ }^{*}$, Carolin Wohlfarth, Michael R Hughes, Kelly M McNagny \\ From AllerGen NCE Inc.'s Fifth Annual Research Conference: Innovation from Cell to Society \\ Québec City, QC, Canada. 7-9 February 2010
}

\section{Objective/purpose}

Eosinophil infiltration of gut tissue plays a key role in the pathogenesis of inflammatory bowel diseases (IBD), such as ulcerative colitis. Using a model of allergic asthma, we previously demonstrated that eosinophil migration requires surface expression of the sialomucin CD34, and that $C d 34$ deletion dampens asthmatic responses in mice. Since CD34 is critical for eosinophil migration, we investigated a role for CD34 in the migration of inflammatory cells into the colon using a mouse model of IBD.

\section{Methods}

To induce ulcerative colitis, we treated animals with 3.5\% dextran sodium sulfate (DSS) and monitored the appearance of clinical symptoms including weight loss, rectal bleeding and diarrhea. Mice were sacrificed after eight days of treatment and we measured colon length, enumerated hematopoietic lineage subsets infiltrating gut tissue by flow cytometry and prepared colon sections for histology to determine the severity of gut pathology. In order to determine the significance of CD34 expression on hematopoietic cells in the development and progression of IBD, we reconstituted wild type mice with $C d 34^{-/-}$ bone marrow to generate chimeras.

\section{Findings}

We found that $C d 34^{--}$mice are highly resistant to DSSinduced IBD with significantly less weight loss and colon shortening than wildtype controls. Histological analysis of $\mathrm{Cd} 34^{-/-}$colons revealed less crypt loss, less tissue infiltrate, reduced tissue ulceration and overall reduced disease severity. We found that approximately

\footnotetext{
* Correspondence: smaltby@br.ubc.ca

The Biomedical Research Centre, University of British Columbia, 2222 Health Sciences Mall, Vancouver, BC, V6T 1Z3, Canada
}

$40 \%$ of the infiltrating blood cells are eosinophils and peripheral eosinophil levels are reduced following disease induction. Intriguingly, eosinophils harvested from the colon express high levels of CD34 and represent the majority of $\mathrm{CD}_{3} 4^{+}$cells within inflamed gut tissue. Protection from DSS-induced IBD is largely recapitulated in mice reconstituted with $\mathrm{Cd} 34^{-/-}$bone marrow, demonstrating the requirement for $\mathrm{CD} 34$ expression on hematopoietic cells in mucosal inflammation.

\section{Deliverables and relevance}

Our findings demonstrate a key role for CD34 on hematopoietic cells in the pathology of ulcerative colitis. Gut eosinophils express high levels of CD34 and, similar to our findings in allergic asthma, we demonstrated that CD34 is required for optimal eosinophil migration in vivo and Cd34 deletion results in decreased gut inflammation during IBD. Taken together, our findings highlight CD34 as a potential therapeutic target for IBD treatment and suggest that therapies targeting CD34 may be sufficient to impair eosinophil infiltration into the colon.

\section{Acknowledgements \\ The research was funded by the AllerGen Network Centre of Excellence (3.14). SM and MRH hold CIHR and Hearth \& Stroke Transfusion Science Fellowships from the Centre for Blood Research (UBC). Kelly McNagny is a Michael Smith Foundation Scholar (Senior) and Centre for Blood Research Member.}

Published: 26 November 2010

doi:10.1186/1710-1492-6-S3-P23

Cite this article as: Maltby et al:: CD34 is required for the infiltration of inflammatory cells into the mouse colon during DSS-induced colitis. Allergy, Asthma \& Clinical Immunology 2010 6(Suppl 3):P23. 